\title{
Collision carcinoma at the esophagogastric junction
}

\author{
Naohiro Washizawa ${ }^{1}$, Kazuo Kobayashi ${ }^{1}$, Hajime Kase ${ }^{1}$, Natsuki Tokura ${ }^{1}$, Tomohiko Gotoh ${ }^{1}$, Iwao Iwasaki ${ }^{1}$, \\ and SHIRo TSUJimoto ${ }^{2}$ \\ ${ }^{1}$ First Department of Surgery, Toho University School of Medicine, 6-11-1 Ohmori Nishi, Ohta-ku, Tokyo 143-8541, Japan \\ ${ }^{2}$ Department of Pathology, Toho University Ohmori Hospital, Tokyo, Japan
}

\begin{abstract}
:
A true collision carcinoma at the esophagogastric junction is rare. In this article, we report colliding double primary cancers of the esophagus and the stomach in a 68-year-old man and discuss this entity. Pathological analysis after total gastrectomy and partial esophagectomy showed the following findings. Areas of squamous differentiation were found on the esophageal side of the tumor and were adjacent to normal mucosa, and areas of glandular differentiation were found to the gastric side of the tumor and adjacent to normal mucosa. These two tumors collided at the esophago-cardiac junction, but there was no intermingling. In one lymph node, an independent non-intermingled metastatic adenocarcinoma and squamous cell carcinoma were observed. The pathological findings of this case satisfy rigorous criteria for collision carcinoma.
\end{abstract}

Key words: gastric cancer, esophageal cancer, collision carcinoma

\section{Introduction}

A true collision carcinoma at the esophagogastric junction is rare. In this article, we report a case of double primary cancers of the esophagus and the stomach and discuss these cancers.

\section{Case report}

A 68-year-old man with no history of gastric complaints reported to a neighboring clinic with dysphagia in

Offprint requests to: N. Washizawa

Received on Nov. 1, 1999; accepted on Jan. 26, 2000
August 1991. Both a circular tumor in the lower esophagus and an irregular elevated lesion in the gastric cardia were discovered by upper gastrointestinal endoscopy. Later, a diagnosis of moderately differentiated adenocarcinoma was made by biopsy. The patient was referred to our hospital in September 1991 for further examination of the lower esophageal tumor. He was not taking any medication and had no history of smoking or excessive alcohol consumption. There were no abnormal abdominal findings; however, there was mild anemia in the palpebral conjunctiva.

Upper gastrointestinal endoscopy attempted in our hospital was not successful, as the endoscope could not pass through an esophageal stricture caused by the circular tumor (Fig. 1). A gastrointestinal series showed a narrowing $4 \mathrm{~cm}$ in length at the lower end of the esophagus, with mucosal irregularity and an irregular filling defect in the gastric cardia (Fig. 2). However, these findings were insufficient for diagnosis. Chest and abdominal plain radiographs were normal. Abdominal computed tomography (CT) scan and ultrasonography showed no evidence of metastasis. Double cancers of the esophagogastric junction were diagnosed because a squamous cell carcinoma was discovered in a biopsy specimen taken from the irregular friable mucosa in the right anterior wall of the lower esophagus and the diagnosis made by previous biopsy.

The patient underwent total gastrectomy, partial esophagectomy, and combined splenectomy without thoracotomy. Extensive lymphadenectomy of the perigastric, esophageal hiatus, and paraesophageal nodes in the lower thorax, as well as the supradiaphragmatic nodes, was also performed. The gastric tumor had not invaded the serosa, but there was evidence of lymphogenous metastasis around the celiac artery. The esophageal tumor had not invaded the muscularis propria. A Roux-en-Y reconstruction was performed, and the patient was discharged on the 35 th postoperative day. 


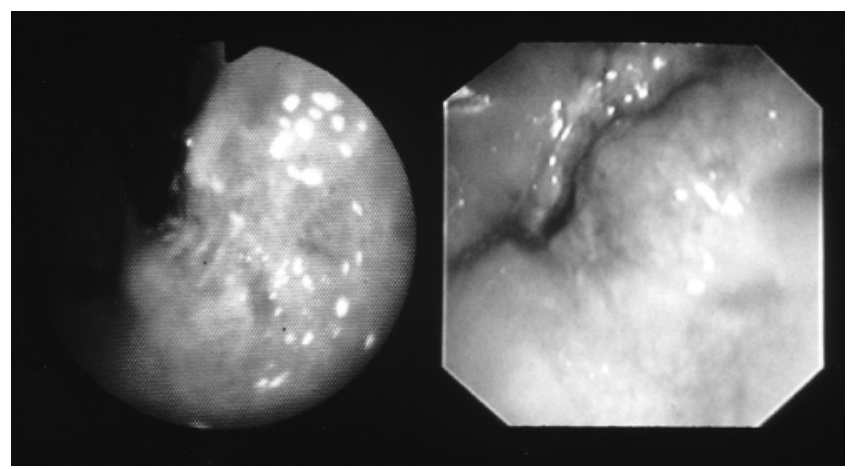

Fig. 1. Endoscopic findings. The endoscope could not pass through an esophageal stricture caused by a circular hard tumor
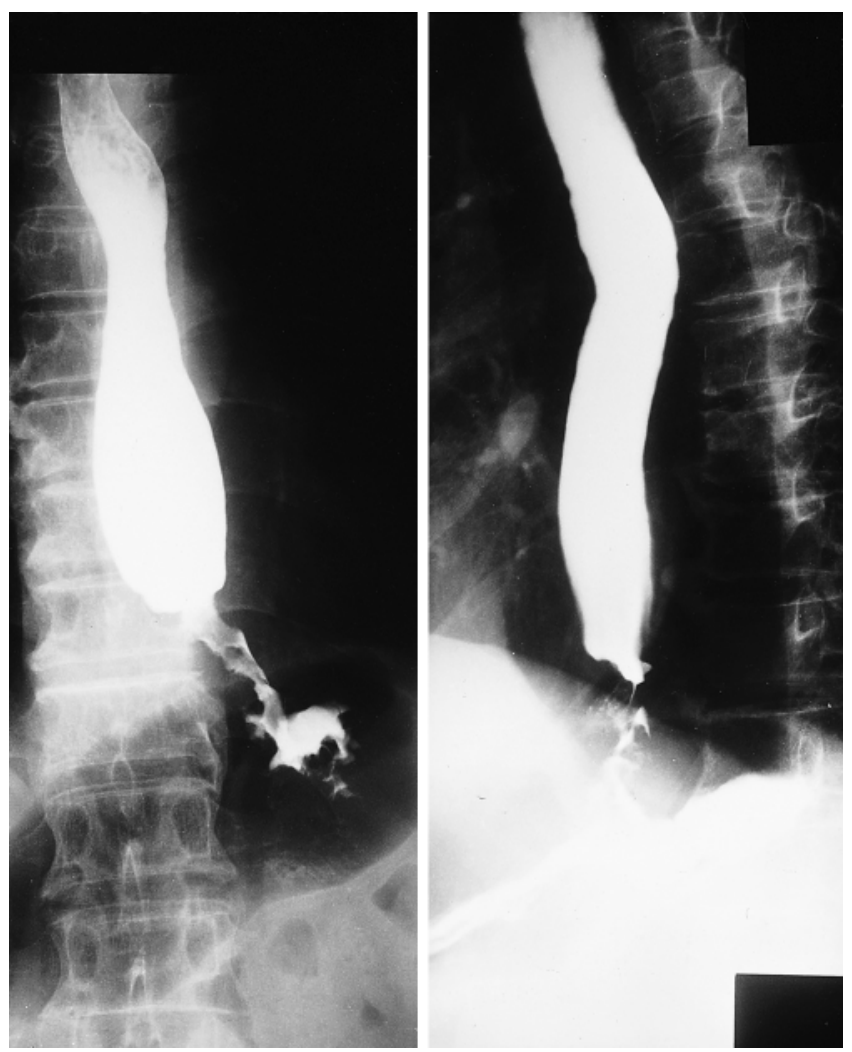

Fig. 2. Preoperative gastrointestinal series shows mucosal irregularity in the lower esophagus and an irregular filling defect in the gastric cardia

Gross examination of the resected specimen revealed ulcerated carcinoma, measuring $5 \times 5.5 \times 2.5 \mathrm{~cm}$, without a capsule, in the gastric cardia and lower esophagus (Fig. 3).

Pathological analysis of the esophageal tumor revealed well differentiated squamous cell carcinoma with cornification invading the muscular layer but not the diaphragm and pleura. The gastric tumor was a moder-

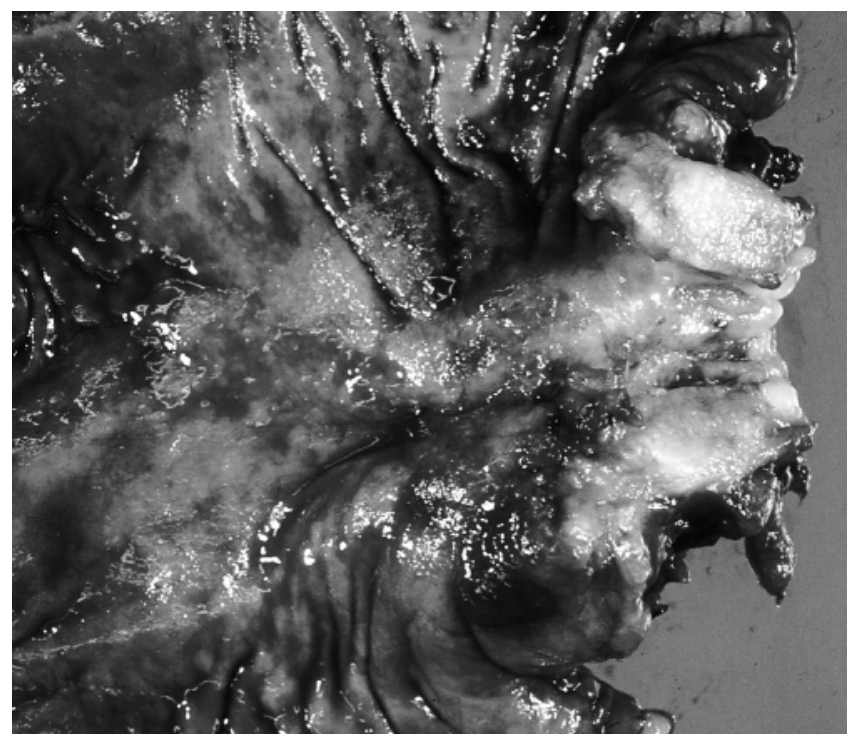

Fig. 3. Gross examination of the resected specimen reveals ulcerated carcinoma without a capsule, in the gastric cardia and lower esophagus

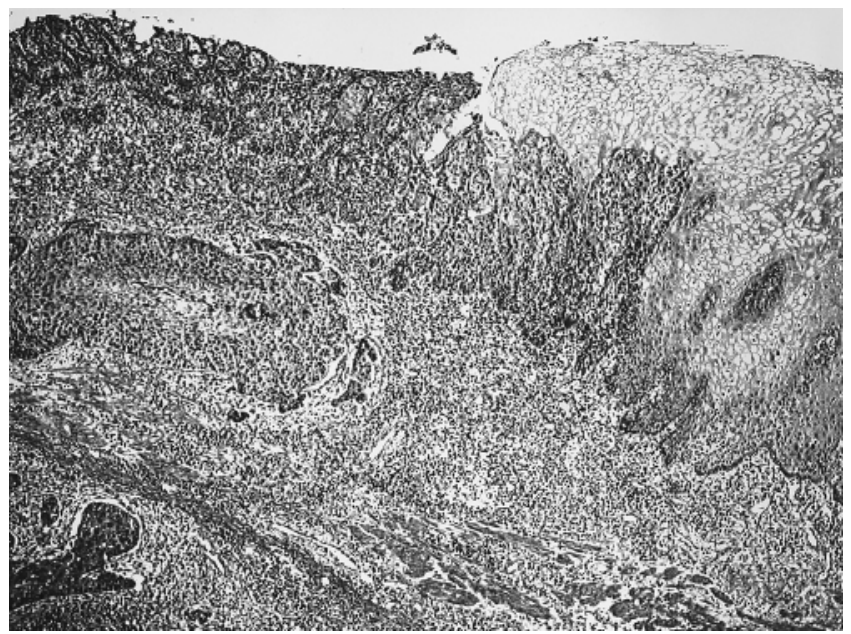

Fig. 4. Photomicrography shows collision of well differentiated squamous cell carcinoma (right side) and moderately differentiated adenocarcinoma (left side). There is no histological transition zone. $\mathrm{H} \& \mathrm{E} \times 400$

ately differentiated adenocarcinoma invading the subserosa. The areas of squamous differentiation were on the esophageal side of the tumor with an epithelial lesion, and were adjacent to normal mucosa. The areas of glandular differentiation were to the gastric side of tumor, with an epithelial lesion, and were adjacent to normal mucosa. These two tumors collided at the esophagocardiac junction, but there was no intermingling (Figs 4, 5). There were tumor emboli in the lymphatics and blood vessels. In one left cardial lymph node, metastatic adenocarcinoma without any squa- 


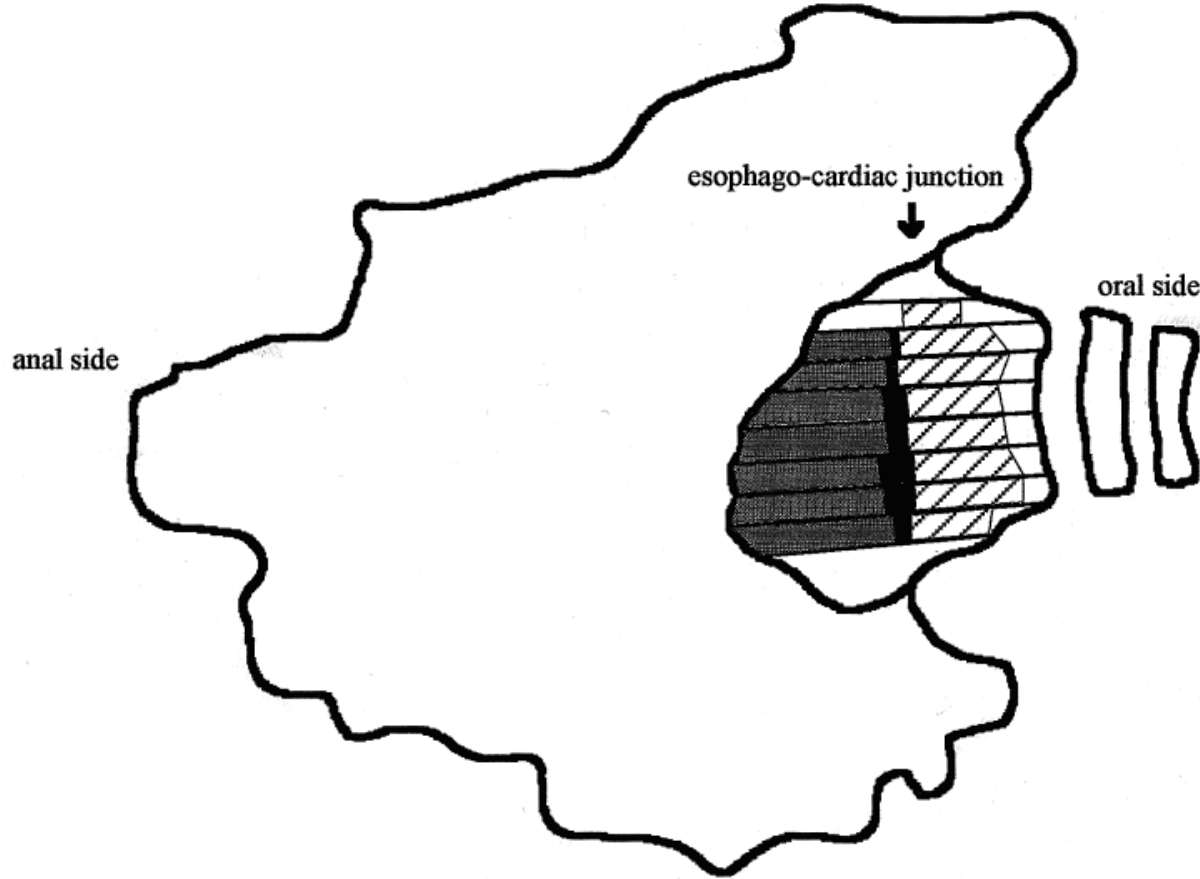

Fig. 5. Schema of the gastric and lower esophageal lesions. The two tumors collided at the esophago cardiac junction. Cross-hatched area, Sqnamous cell carcinoma; black area, collision area; gray area, adenocarcinoma mous component was discovered. In another cardial lymph node, independent non-intermingled metastatic adenocarcinoma and squamous cell carcinoma were observed. A supradiaphragmatic lymph node showed metastatic squamous cell carcinoma.

The patient's immediate postoperative course was uneventful, but he died of peritoneal recurrence of squamous cell carcinoma, in October 1992. An autopsy was not performed, so a detailed pathological report was not possible.

\section{Discussion}

The present case was diagnosed, on the basis of histological evidence, as a collision carcinoma at the esophagogastric junction. The occurrence of colliding esophageal squamous cell carcinoma and gastric adenocarcinoma at the esophagogastric junction is rare. We found seven previous reports of esophagogastric collision tumor in the literature [1-7].

It was noted that combined carcinomas generated from two different histological types of tumors were classified into three groups. (1) Combination tumors that are separated into adenocarcinomas and squamous cell carcinomas by their generation. (2) Composition tumors that consist sarcoma associated with epithelial carcinogenesis. (3) Collision tumors that are generated from two different origins; for example, esophagus and stomach, in which two different tumors form one tumor after colliding with each other.
Meyer [8] defines a collision tumor as the colliding of histologically different tumors from independent primary sites. Wanke [1] and Spagnolo and Heenan [3] insist on the presence of a zone of intermingling or gradual transition. Dodge's [9] criteria include partial topographical separation and little or no evidence of an intermediate histological structure.

In our patient, areas of squamous differentiation were present in normal esophageal epithelium and the carcinoma was highly differentiated. Areas of glandular differentiation were discovered in normal gastric epithelium and the carcinoma was moderately differentiated. Therefore, we believe the two carcinomas arose independently.

Two histologically different tumors had collided without intermingling. Indeed, there was no intermingling of either the primary tumors or of metastases in the left cardial lymph node. Therefore, the pathology findings in this patient satisfy Dodge's criteria.

Collision tumors can be difficult to detect and diagnose [6]. Indeed, the disease in the present patient could not be diagnosed by upper gastrointestinal series or endoscopy. However, the presence of differing findings on pathology reports should raise clinicians' suspicions. If a preoperative diagnosis can be made, the patient's prognosis prognosis may be improved by the selection of preoperative radiotherapy or chemotherapy. Moreover, mediastinal and abdominal lymphadenectomy may be indicated in patients with esophageal and gastric double carcinomas. Clearly, clinicians need to be aware of the possibility of esophagogastric collision carcinoma. 


\section{References}

1. Wanke M. Case report, Collision-Tumor of the cardia. Virchows Arch Abt A Path Anat 1972;357:81-6.

2. Majmudar B, Dillard R, William SP. Collision carcinoma of the gastric cardia. Hum Pathol 1978;9:471-3.

3. Spagnolo DV, Heenan PJ. Collision carcinoma at the esophagogastric junction. Cancer 1980;46:2702-8.

4. Andoh T, Oka Y, Kurokawa S, Morise K, Kurokawa S, Hikabe A, et al. Collision carcinoma at the cardia diagnosed by endoscopy. Report of a case. I to Cho (Stomach and Intestine) 1991;26:313-9.

5. Matsumoto M, Shirao K, Yoshinaka H, Baba M, Fukumoto T, Aikoh T. A case of esophago-residual gastric collision carcinoma. Rinsho Geka (J Clin Surg) 1994;49:787-91.
6. Maemura T, Sueyoshi S, Shida S, Suga K, Fukushima S, Arakawa M. A case of colliding gastric adenocarcinoma and esophageal squamous cell carcinoma at the esophagogastric junction. Nippon Rinsho Geka Igakkai Zasshi (J Jpn Soc Clin Surg) 1997;58:1497-503.

7. Kawada S, Murakami S, Noguchi T, Hashimoto T, Uchida Y, Urabe S. A case of collision tumor with esophageal squamous cell carcinoma and gastric signet ring cell carcinoma at the cardia. Nippon Rinsho Geka Gakkai Zasshi (J Jpn Surg Assoc) 1998;59:2424-7.

8. Meyer R. Beitrag zur Verstandigung uber die Namengebung in der Geschwulstlehrle. Zentralbl Pathol 1919;30:291-6.

9. Dodge OG. Gastro-oesophageal carcinoma of mixed histological type. J Pathol Bacteriol 1961;81:459-71. 